\title{
Database Design on Mechanical Equipment Operation Management System
}

\author{
Zheng Qiu ${ }^{1}$, Wu kaiyuan ${ }^{1}$, Wu Chengyan ${ }^{1}$, Liu Lei ${ }^{2}$ \\ ${ }^{1}$ No.208 Research Institute of China Ordnance Industries, Beijing 102202 \\ ${ }^{2}$ College of Engineering, Bohai University, Jinzhou, 121013
}

Keywords: mechanical equipment; operation management; database design; conceptual design; logical design; physical design

\begin{abstract}
Carrying out mechanical equipment operation management can improve the economic efficiency for enterprises, prevent accidents, ensure production safety, and promote the reform of machinery and equipment maintenance system. Using computer system to manage is the necessary requirements for the information age. For the difficulties of system development, this paper conducted the database design. Following the database design process conducted the conceptual design, logical design and physical design. Conceptual design using the E-R diagram approach, suggested a link between entities and entity; logic design is based on SQL Server, designed the logical structure for all the tables; physical design about storage structures and access methods, including the disk hardware selection, data place on the disk, use the index to improve system performance, configuration parameters and other aspects design.
\end{abstract}

\section{Introduction}

Mechanical equipment operation management with the life cycle of mechanical equipment for industrial enterprises as the object of the research, pursuit the most economical life-cycle costs of machinery and equipment and the highest overall effectiveness as the goal, scientifically research the comprehensive technical, economic and regulatory factors. Mechanical equipment operation management task is to ensure provide the best technology and equipment, so that production activities based on the best material and technical basis. Manage well, use well and repaired mechanical equipment, with minimal maintenance costs, obtained the maximum performance. The general trend of mechanical equipment operation management is modernization, namely Mechanical equipment operation management integration, full of computerization, networking and intelligent.

Great significance for developing equipment operation management in three aspects [1-3]: First, increase economic efficiency. With modern industrial development and science and technology, machinery and equipment toward large-scale, continuous, high-speed and automation direction, large machinery and become a modern large-scale production of key equipment, once the downtime will result in discontinued, causing huge economic loss. Equipment operation and management can reduce accident losses and extend the maintenance period, bringing considerable economic benefits; the second is to prevent accidents and ensure safety in production. Machinery and equipment during operation without failure is impossible, absolutely safe and reliable equipment does not exist, the best method is to detect anomalies and failures to grasp the development trend of equipment to take timely and appropriate control measures to prevent major accidents ensure personal and equipment safety; third is to promote the reform of the mechanical equipment maintenance system. Machinery and equipment maintenance system has gone through three stages, namely corrective maintenance, preventive maintenance and predictive maintenance. Corrective maintenance is also known as breakdown maintenance, equipment failure before maintenance. Preventive maintenance is also known as regular maintenance, according to a predetermined time interval or maintenance cycle, maintenance, adjustment and replacement of spare parts. Predictive maintenance is also known as condition monitoring maintenance, inspection and test equipment for a planned way, to determine the health status. This repair method can bring significant economic benefits. The regular 
maintenance to the predictive maintenance, saving maintenance costs, reducing maintenance time and increases the mechanical equipment uptime, increase productivity, resulting in huge economic benefits.

Mechanical equipment operation management can keep abreast of the operational status of equipment, it is determined whether abnormal or fault status of Mechanical equipment operation control, predict trends. For the fault has been formed to analyze the severity of the fault, the fault type, location and cause of failure, to take effective control measures as soon as possible. Through the Mechanical equipment operation and management, access to Mechanical equipment running status feature information, grasp the changes in the operating status Mechanical equipment, Mechanical equipment according to the state to decide whether to require maintenance, saving the cost of Mechanical equipment into the maintenance area. At the same time maximize the use of machinery and equipment; improve the operating efficiency of machinery and equipment. Development of mechanical equipment operation management system is important. In this paper, the database design is an important part of software development, to improve the efficiency and quality of system development has great significance.

\section{Database Design Process}

The basic database design process can be divided into four steps, namely needs analysis, conceptual design, logical design and physical design, it is shown in Fig. 1 [1].

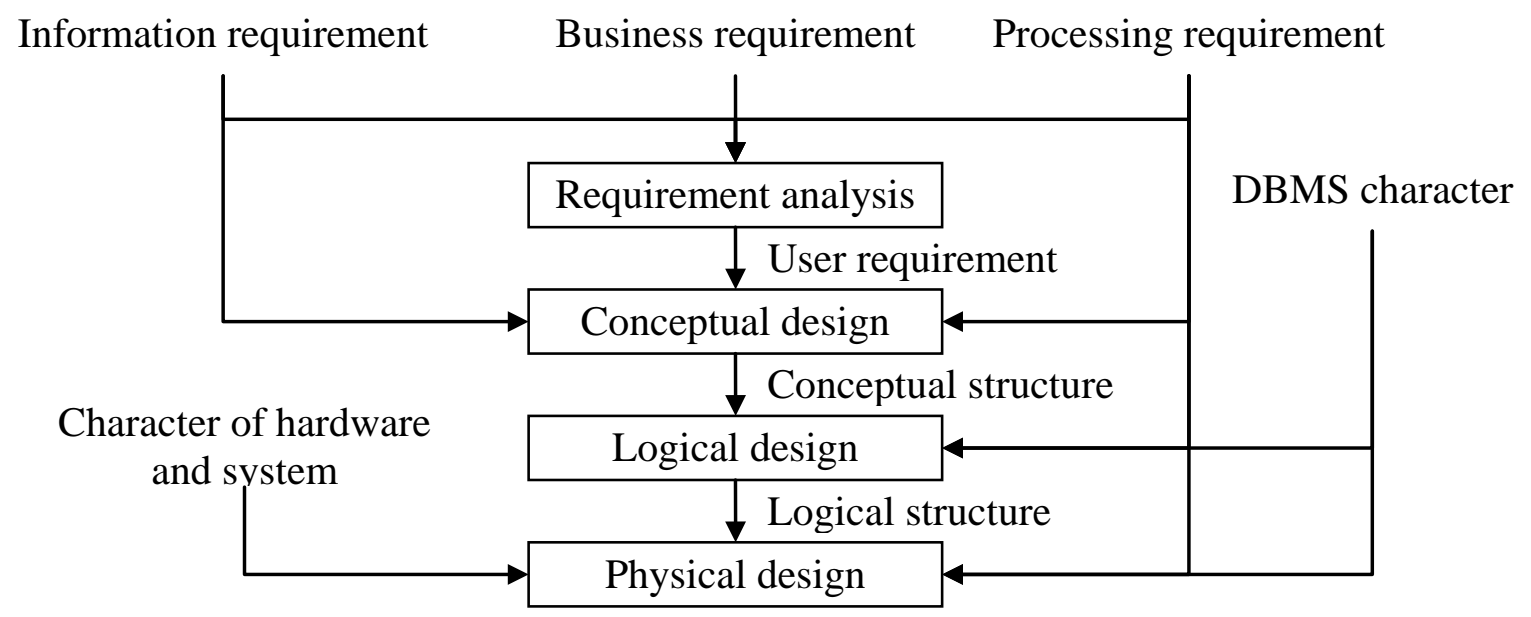

Fig. 1. Steps of database design

The main task of needs analysis was to investigate and analyze the user's business activities, information and processing requirements, as well as a variety of constraints, form database design requirements specification. Conceptual design task is conceptual design of the database structure, determined from the point of view of different users to see outside pattern and the outer mode integrated into the overall pattern. Logical design is the overall pattern of conceptual design was converted into the selected database model, and optimization. The task is to determine the physical design within the schema of the database. Development methodology and information system database design methodology employed there is a close relationship, but also with the database models adopted relevant.

\section{Conceptual Design}

The user needs get in the needs analysis is abstracted information structure, namely conceptual model is the conceptual design process [4]. Conceptual design goal is to reflect the organization's overall information needs of database conceptual structure; in accordance with a specific method abstract data model does not rely on any particular machine, that conceptual model. The concept model is the information structure of each user of common concern throughout the organization, 
you must be independent of any computer data model [5], a conceptual model is simple, clear and easy for users to understand, is the language of communication between the user and the database designer [6]. Conceptual design is modeled on the world of information, common conceptual model ER model, the results of conceptual design for the system is shown in Fig. 2.

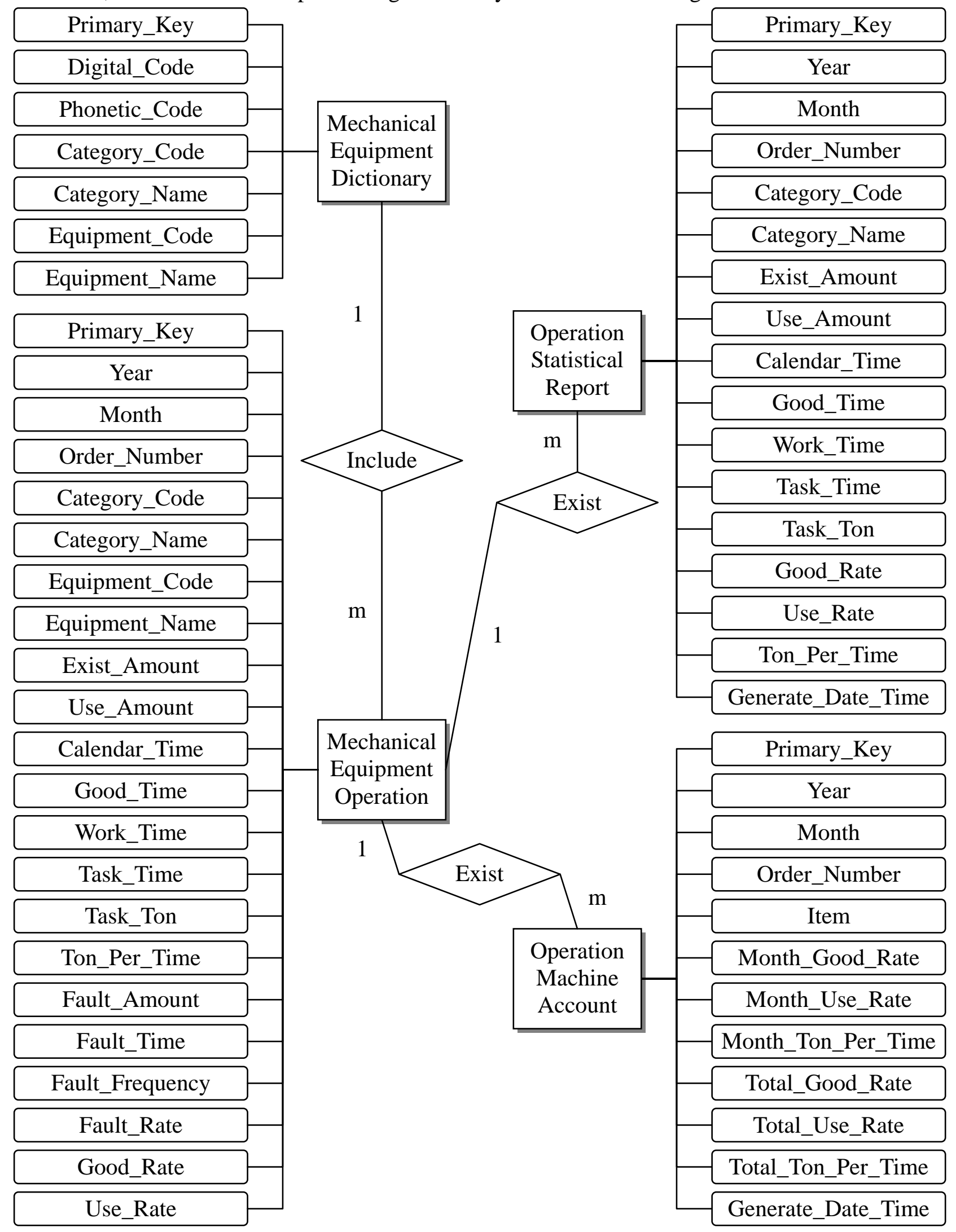

Fig. 2. Conceptual design on mechanical equipment operation management system 


\section{Logical Design}

From conceptual design to the logical database design is an important part of the design process. ER model concept design phase was to reflect the needs of the business data model, regardless of the specific data model and DBMS. In order to establish the required database user, conceptual models need to be converted to a specific DBMS support data model. The task is to convert a conceptual model for a particular DBMS process supported by the data model; we need to consider the characteristics for specific data model and DBMS performance [7-9]. Logical design results based on SqlServer are shown in Table 1 below.

Table 1. Logical structure design on teaching management system

\begin{tabular}{|c|c|c|c|c|c|c|c|}
\hline \multicolumn{4}{|c|}{ Mechanical_Equipment_Dictionary } & \multicolumn{4}{|c|}{ Operation_Statistical_Report } \\
\hline No & Field name & Data type & Byte & No & Field name & Data type & Byte \\
\hline 1 & Primary_Key & varchar & 20 & 1 & Primary_Key & varchar & 20 \\
\hline 2 & Digital_Code & smallint & 2 & 2 & Year & char & 4 \\
\hline 3 & Phonetic_Code & varchar & 4 & 3 & Month & char & 2 \\
\hline 4 & Category_Code & varchar & 3 & 4 & Order_Number & Smallint & 2 \\
\hline 5 & Category_Name & varchar & 20 & 5 & Category_Code & varchar & 3 \\
\hline 6 & Equipment_Code & varchar & 10 & 6 & Category_Name & varchar & 20 \\
\hline 7 & Equipment_Name & varchar & 50 & 7 & Exist_Amount & int & 4 \\
\hline \multicolumn{4}{|c|}{ Mechanical_Equipment_Operation } & 8 & Use_Amount & int & 4 \\
\hline No & Field name & Data type & Byte & 9 & Calendar_Time & int & 4 \\
\hline 1 & Primary_Key & varchar & 20 & 10 & Good_Time & int & 4 \\
\hline 2 & Year & char & 4 & 11 & Work_Time & int & 4 \\
\hline 3 & Month & char & 2 & 12 & Task_Time & int & 4 \\
\hline 4 & Order_Number & Smallint & 2 & 13 & Task_Ton & int & 4 \\
\hline 5 & Category_Code & varchar & 3 & 14 & Good_Rate & decimal & 7,2 \\
\hline 6 & Category_Name & varchar & 20 & 15 & Use_Rate & decimal & 7,2 \\
\hline 7 & Equipment_Code & varchar & 10 & 16 & Ton_Per_Time & decimal & 7,2 \\
\hline 8 & Equipment_Name & varchar & 50 & 17 & Generate_Date_Time & datetime & 8 \\
\hline 9 & Exist_Amount & int & 4 & \multicolumn{4}{|c|}{ Operation_Machine_Account } \\
\hline 10 & Use_Amount & int & 4 & No & Field name & Data type & Byte \\
\hline 11 & Calendar_Time & int & 4 & 1 & Primary_Key & varchar & 20 \\
\hline 12 & Good_Time & int & 4 & 2 & Year & char & 4 \\
\hline 13 & Work_Time & int & 4 & 3 & Month & char & 2 \\
\hline 14 & Task_Time & int & 4 & 4 & Order_Number & Smallint & 2 \\
\hline 15 & Task_Ton & int & 4 & 5 & Item & varchar & 30 \\
\hline 16 & Ton_Per_Time & decimal & 7,2 & 6 & Month_Good_Rate & decimal & 7,2 \\
\hline 17 & Fault_Amount & int & 4 & 7 & Month_Use_Rate & decimal & 7,2 \\
\hline 18 & Fault_Time & int & 4 & 8 & Month_Ton_Per_Time & decimal & 7,2 \\
\hline 19 & Fault_Frequency & decimal & 7,2 & 9 & Total_Good_Rate & decimal & 7,2 \\
\hline 20 & Fault_Rate & decimal & 7,2 & 10 & Total_Use_Rate & decimal & 7,2 \\
\hline 21 & Good_Rate & decimal & 7,2 & 11 & Total_Ton_Per_Time & decimal & 7,2 \\
\hline 22 & Use_Rate & decimal & 7,2 & 12 & Generate_Date_Time & datetime & 8 \\
\hline
\end{tabular}




\section{Physical Design}

Physical design is based on logical design, select the appropriate storage structures and access methods for each of the relational model, making the transaction on the database can be efficiently run. Physical structure design depends on the specific database management system. For Microsoft SQL Server the physical design as follows.

(1) Disk hardware selection. There are many hard drive performance parameters, but for access to the hard drive to select the database mainly from the capacity, speed, cache and other aspects to consider. If you choose a dedicated database server, consider the disk redundant array of devices.

(2) The data is placed on the disk. If the data is less, the data can be placed on file; if large amount of data, it is necessary to place on the file group. File group consists of more than one file in a different physical disk composition, can improve query performance.

(3) Using the index to improve performance. When there is no index on the column is accessed, SQL execution time is mainly consumed in the loading and data matching table data, you need to consume a lot of CPU costs and IO costs [10].

(4) Configuration parameters to make the database to run well. First, the use of memory configuration options min server memory, max server memory, max worker threads, index create memory, min memory per query and so optimize server performance; second is to use I/O configuration options to optimize server performance recovery interval.

\section{Conclusion}

With the development of information technology, the method that computer process data undergo the profound changes, SQL Server is a widely used database management system with client/server architecture, can meet business environments require different types of database solutions. Has many significant advantages, such as usability, scalability for distributed organizations, the data warehouse for decision support, closely associated with other server software, and so on. This paper based on SQL Server to design, for system development to provide a viable solution, and for improving the mechanical equipment operation management level has important.

\section{References}

[1] Z. J. He, H. D. Cao, Y. Y. Zi, et al, "Developments and Thoughts on Operational Reliability Assessment of Mechanical Equipment," Journal of Mechanical Engineering, vol. 50, no. 2, pp. 171-186, 2014.

[2] M. J. Chen, "Maintenance and management of mechanical equipment from the perspective of economic operation," China Management Informationization, vol. 16, no. 10, pp. 107-108, 2013.

[3] L. Z. Miao, "Management measures for safety operation of mechanical equipment," Journal of Henan Science and Technology, vol. 48, no. 16, pp. 77-78, 2013.

[4] Baidu Encyclopedia, "Conceptual Structure Design," http://baike.baidu.com/view/1638403.htm, 2016-1-10.

[5] L. Zhang, L. Ma, "Database Design," Journal of Anyang Institute of Technology, vol. 6, no. 4, pp. 76-79, 2007.

[6] Z. Y. Duan, "Database Design Method," Journal of Nanchang College of Education, vol. 20, no. 4, pp. 84-86, 2005.

[7] Y. Zhang, "Eliminating Process of Formalization in Logical Database Design," Computer Systems \& Applications, vol. 22, no. 6, pp. 179-181, 2013.

[8] doc88, "Logical structure design," http://www.doc88.com/p-117617790552.html, 2015-2-10.

[9] H. M. Chen, "Logical structure design of database," Fujian Computer, vol. 28, no. 10, pp. 214-215, 2012.

[10] J. S. Yang, C. Zhang, "Effect of index on SQL execution cost," Information communication, vol. 27, no. 1, pp. 136-137, 2013. 